\title{
Estimativa de porosidade e permeabilidade através de imagens digitais de rochas artificiais
}

Santos, R. A. A*., Galvão, I. L. G., Segundo, F. R. D. P., Xavier Jr., M. M., Cabral, F. A. O. Departamento de Geofísica, Universidade Federal do Rio Grande do Norte.

Copyright 2014, SBGf - Sociedade Brasileira de Geofísica

Este texto foi preparado para a apresentação no VI Simpósio Brasileiro de Geofísica, Porto Alegre, 14 a 16 de outubro de 2014. Seu conteúdo foi revisado pelo Comitê Técnico do VI SimBGf, mas não necessariamente representa a opinião da SBGf ou de seus associados. É proibida a reprodução total ou parcial deste material para propósitos comerciais sem prévia autorização da SBGt.

\section{Resumo}

Este trabalho teve como objetivo realizar uma estimativa digital da porosidade e permeabilidade de rochas artificiais usando a técnica de segmentação de imagens digitais de microtomografia de raios- $X$ e de lâminas petrográficas. Trata-se de uma pesquisa com abordagem quantitativa, realizada em amostras de rochas artificiais obtidas através da sinterização de "esferas de vidro". A técnica de imagens digitais consiste na visualização da estrutura interna da amostra de uma forma não destrutiva permitindo assim uma análise mais completa da porosidade e permeabilidade das amostras. Em seguida, os resultados simulados pelas imagens digitais foram comparados com as medidas experimentais de porosidade e permeabilidade, no intuito de avaliar a confiabilidade de ambas as técnicas de imagens. O software usado na visualização e processamento das imagens foi o IMAGO.

\section{Introdução}

A análise de imagem digital é uma técnica inovadora que visa melhorar o entendimento sobre a geometria dos espaços porosos de uma amostra de rocha (plug). Essa nova metodologia simula e determina características importantes da rocha, tendo como exemplo, a determinação da porosidade, da permeabilidade, da distribuição do tamanho do poro e principalmente da reconstrução de um modelo tridimensional. O desenvolvimento tecnológico usado na criação de equipamentos, hardwares e softwares vem possibilitando uma maior rapidez na aquisição e no processamento das imagens (CUNHA et al, 2012).

Para a aquisição das imagens digitais foram utilizadas amostras de rochas artificiais em forma de plugues cilíndricos (Figura 1) (SEGUNDO, 2014). Essas rochas artificiais são formadas por "esferas de vidro" contendo faixas de diâmetro na escala de micrômetros a milímetros.

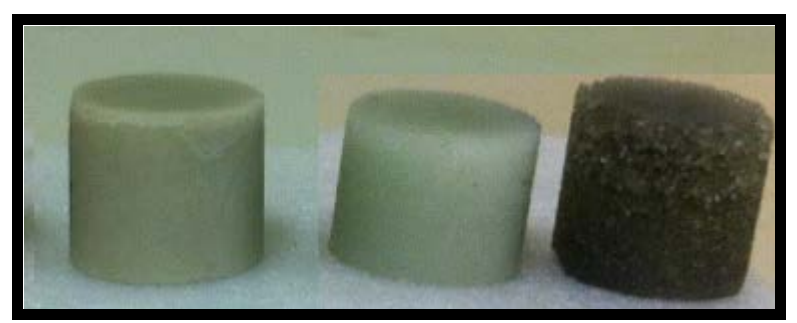

Figura 1. Rochas artificiais: (RA_0,125), (RA_0,355), (RA_1,00).
As amostras foram rotuladas pela sigla RA (rocha artificial) e numeradas de acordo com os diâmetros das esferas de vidro usadas na sua fabricação, por exemplo, RA_0,125 (rocha artificial, esferas com diâmetro de $0, \overline{1} 25$ milímetros).

\section{Metodologia}

Para a análise e processamento das imagens de tomografia e petrografia das rochas artificiais foi usado o software IMAGO (SILVA, 2011, ROCHA et al, 2005)). Os procedimentos realizados para a obtenção das medidas petrofísicas a partir da análise de imagens digitais são feitos de maneira similar para todos os materiais de uma maneira geral, ou seja, não importa o tipo de material de que é feita a amostra ou rocha empregada no estudo. Sendo assim os procedimentos básicos são: obtenção da amostra, aquisição das imagens digitais e processamento (aplicação de filtros, delimitação da área da imagem para o estudo, resolução linear, segmentação ou binarização, geometria da microestrutura, calculo da porosidade, calculo da permeabilidade, reconstrução 3D).

\section{Resultados e Discussão}

O processo de segmentação das imagens foi realizado com 0 auxilio de três tipos de metodologias disponibilizadas pelo IMAGO, sendo estas a Gray Thresholding, a RGB Thresholding e a HSI Thresholding, onde a primeira foi usada apenas na imagem de microtomografia e as duas últimas utilizadas na binarização das imagens de lâmina petrográfica (DE GASPERI,1999).

Antes da binarização, todas as imagens foram tratadas como a aplicação do filtro espacial passa baixa no intuito de eliminar o máximo de "ruído" causado no momento da aquisição das fotos, alem modelar de uma maneira satisfatória as bordas da interfase poro-grão. Entretanto, a maior dificuldade no momento da segmentação, esta no fato de determinar a melhor distinção do que é poro e do que é grão. Uma ilustração do processo de binarização das imagens está mostrada nas figuras 2 a 5.

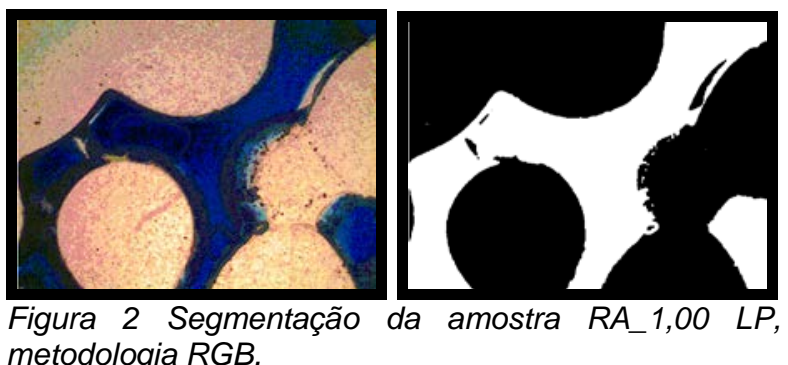




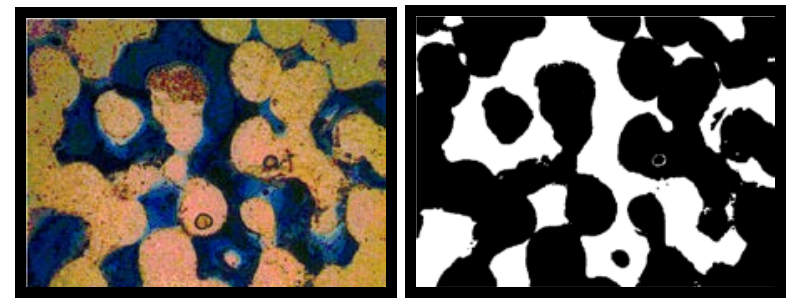

Figura 3 Segmentação da amostra RA_0,355 LP, metodologia RGB.
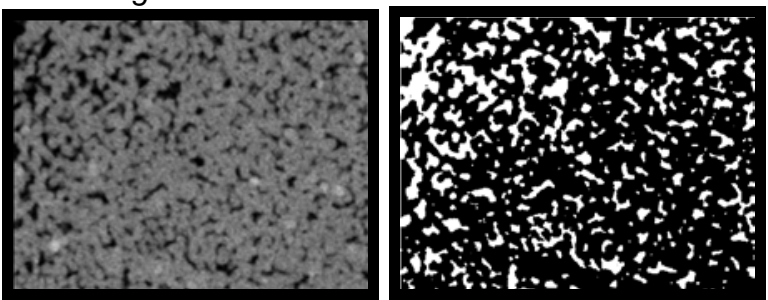

Figura 4. Segmentação da amostra $R A-0.355 T$ metodologia Gray.
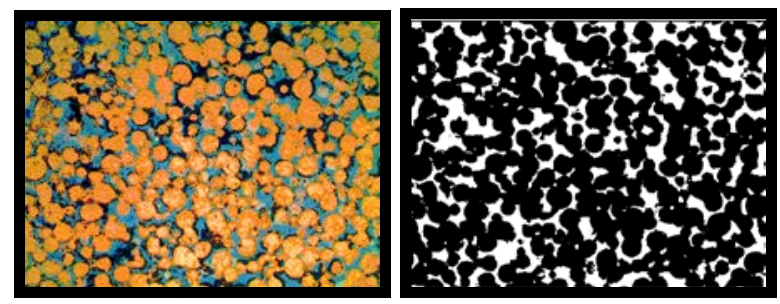

Figura 5. Segmentação da amostra RA_ 0,125 LP metodologia HSI.

A porosidade alcançada com o auxilio do software IMAGO (Tabela 1), obteve valores considerados bastante satisfatórios em relação aos resultados experimentais apresentando um erro entre 7\% a 34\% nos valores de porosidade (GALVÃO, 2014). Tais valores podem ser explicados pelo grau de seleção e arredondamento das partículas que formam a amostra, o que facilitou o processo de binarização das imagens.

Tabela 1. Valores de Porosidade obtidos pelo IMAGO e por petrofísica de laboratório. * $L P$ refere-se a imagem de lâmina petrográfica $e$ * $T$ refere-se a imagem de tomografia de raios- $X$.

\begin{tabular}{|c|c|c|}
\hline \multirow{2}{*}{ Amostra } & \multicolumn{2}{|c|}{ Porosidade (\%) } \\
\cline { 2 - 3 } & Petrofísica & IMAGO \\
\hline RA_0,125 LP & 36.679 & 33.84 \\
\hline RA_0,355 LP* & 24.889 & 33.258 \\
\hline RA_0,355 T* & 24.889 & 20.327 \\
\hline RA_1.000 LP & 23.784 & 30.830 \\
\hline
\end{tabular}

Na amostra RA_0.355 foi possível analisá-la utilizando tanto as imagens de lâminas petrográficas quanto as imagens de microtomografia de raio- $X$, sendo que os resultados descritos na tabela 1 apontam a imagem tomográfica como sendo a estimativa de maior acerto, em torno de $81 \%$, se comparada com a lâmina que ficou com um acerto em torno de $66 \%$, isso para os valores de porosidade obtidos na mesma amostra. Entretanto, uma possível razão para explicar esse contraste de resultados, é a maneira como ambas as imagens foram binarizadas, ou seja, a imagem de microtomografia teve um grau de amostragem maior (LOPES et al, 2011).
Para as estimativas de permeabilidade, foram utilizados os métodos booleano de gás em rede, Lattice Gas (L.G.), e o Fast K, ambos disponibilizados pelo software IMAGO. Os valores descriminados na tabela 2 mostram que o IMAGO não apresentou resultados nem um pouco semelhantes com as medidas petrofísicas, extrapolando erros acima de $100 \%$. Um dos fatores que podem justificar esses valores é a resolução (tamanho do pixel) em que foi utilizado nas imagens. Outro fator que pode ter influenciado nas medidas de permeabilidade são os parâmetros de entrada usados na reconstrução 3D, onde o alcance de correlação que indica a ordenação dos poros na estrutura exibiu altos valores o que causa uma grande variação nos valores obtidos pelo modelo.

Tabela 2. Valores de Permeabilidade obtidos pelo IMAGO e por petrofísica de laboratório. * $L P$ refere-se a imagem de lâmina petrográfica $e$ * $T$ refere-se a imagem de tomografia de raios- $X$.

\begin{tabular}{|l|c|c|c|}
\hline \multirow{2}{*}{ Amostra } & \multicolumn{3}{|c|}{ Permeabilidade (mD) } \\
\cline { 2 - 4 } & Petrofísica & $\begin{array}{l}\text { IMAGO } \\
\text { (L.G.) }\end{array}$ & $\begin{array}{l}\text { IMAGO } \\
\text { (fast K) }\end{array}$ \\
\hline RA_0,125 LP & 33240.00 & 756.52 & 375.86 \\
\hline RA_0,355 LP* & 124965.00 & 39.74 & 360.43 \\
\hline RA_0,355 T* & 124965.00 & 12240.29 & 11495.73 \\
\hline RA_1.000 LP & 332407.00 & 58.32 & ---------- \\
\hline
\end{tabular}

Para a amostra RA 0.355 o tipo de imagem, seja ela de lâmina ou de tomografia, não influenciou no momento do resultado final, demonstrando que ambas obtiveram valores exagerados de permeabilidade.

\section{Conclusões}

Para as estimativas de porosidade, a metodologia de imagens tomográficas obteve os melhores resultados se comparado as imagens de lâminas petrografias, entretanto, há uma necessidade na realização aprofundar a pesquisa envolvendo ambas as técnicas de imagens para confirmar tais resultados de uma maneira mais ampla, já que nesse trabalho só foi possível a comparação em uma única amostra (RA 0,355). Vale salientar que tratamos com rocha artificial o que facilitou o processo de binarização e que não ocorre normalmente com rochas reais. Já o software usado no processamento, o IMAGO, atendeu de maneira satisfatória os cálculos de porosidade, demonstrando resultados semelhantes com as medidas de porosidade realizadas experimentalmente.

Para as estimativas de permeabilidade, o IMAGO apresentou resultados extremamente diferentes das medidas adquiridas experimentalmente, isso ocorreu tanto nas imagens petrografias quanto nas imagens tomográficas, admitindo a resolução (tamanho do pixel) em que foi feito as imagens digitais, como possível motivo pela enorme discrepância entre os valores experimentais e simulados. Outro possível motivo, sendo este agora ligado diretamente ao IMAGO, está relacionado a reconstrução do modelo $3 \mathrm{D}$ das imagens. Portanto, tona-se necessário uma maior analise nos parâmetros usados na reconstrução 3D, bem como a utilização de outros softwares com a função de comparar os resultados alcançados. 
Os resultados obtidos a partir desse trabalho com imagens digitais poderão ajudar no desenvolvimento de softwares relacionado a estimativas de grandezas petrofisicas que está sendo realizado no departamento de matemática da UFRN.

\section{Agradecimentos}

Ao Professor José Agnelo Soares (UFCG) pela contribuição nas medidas de porosidade. A professora Marcela Marques Vieira (UFRN) pela obtenção das imagens de lâminas delgadas. Ao Professor Leonardo Borghi (UFRJ) pelo apoio na obtenção das imagens tomográficas. A ANP pelo apoio financeiro ao projeto.

\section{Referências}

CUNHA, A.R., MOREIRA, A.C., KRONBAUER, D.P., MANTOVANI, I.F., FERNANDES, C.P. 2012. Determinação de propriedades petrofísicas de rochas via simulação. Um caminho interdisciplinar Universidade Federal de Santa Catarina Florianópolis.

SEGUNDO, F.R.D.P, 2014. "Síntese de rochas artificiais através da sinterização de esferas de vidro", Relatório de graduação em geofísica - Universidade Federal do Rio Grande do Norte - Depto. de Geofísica, Natal.

SILVA, L.R.D, 2011 "Analise computacional de imagens de lâminas delgadas na predição das propriedades porosidade e permeabilidade da porção aflorante de rochas do arquifero Açu-RN", Relatório de graduação em geofísica - Universidade Federal do Rio Grande do Norte - Depto. de Geofísica, Natal.

ROCHA, W.R.D,. APPOLONI, C.R,. OLIVEIRA, L.F,. FERNANDES, C.P,. LOPES, R.T,. 2005. Análise Microestrutural de Cerâmicas Porosas de SiC por Transmissão de Raios Gama e Microtomografia de Raios X - Universidade Estadual de Londrina - Depto de Física/ Centro de Ciências Exatas

DE GASPERI, P.M.S, 1999. "Estimativa de Propriedades Petrofísicas através da Reconstrução 3D do Meio Poroso a partir da Análise de Imagens", Dissertação de Mestrado - Universidade Estadual de Campinas - Faculdade de Engenharia Mecânica Depto. de Engenharia de Petróleo, Campinas.

GALVÃO, I.L.G, 2014 “ Estudos das Propriedades Petrofísicas de Rochas Artificiais", Relatório de graduação em geofísica - Universidade Federal do Rio Grande do Norte - Depto. de Geofísica, Natal.

LOPES, A.P,. FIORI, A.P,. REIS NETO, J.M,. MARCHESE, C,. VASCONCELLOS, E.M.G,. TRZASKOS, B,. ONISHI, C.T,. PINTO-COELHO, C.V,. SECCHI, R,. SILVA G.F,. 2012. Análise tridimensional de rochas por meio de microtomografia computadorizada de raios $\mathrm{X}$ integrada à petrografia. 